greater; however in $27 \%$ (7) cases this was static, and in $11 \%$ (3) bowel wall thickness was greater. Total disease burden was greater in 2 patients. Complete radiological remission was demonstrated in only 2 patients.

Conclusion Response of Crohn's disease to ATT is well documented, and we are able to demonstrate quantifiable interval improvement using MRE. Recording the disease burden by way of stricture length and bowel wall thickness is a mode of measuring MRE response to treatment in Crohn's disease and may be used for disease reassessment as required by NICE.

Disclosure of Interest None Declared.

\section{PTH-093 EFFICACY OF METHOTREXATE IN ULCERATIVE COLITIS: A DISTRICT GENERAL HOSPITAL EXPERIENCE}

doi:10.1136/gutjnl-2013-304907.580

1."R Basuroy, 'H E Johnson, 'T Hollingworth, 'S A Weaver, 'S D McLaughlin. 'Department of Gastroenterology, Royal Bournemouth \& Christchurch Hospitals NHS Foundation Trust, Bournemouth, UK

Introduction Uncontrolled studies have reported methotrexate (MTX) to be efficacious in patients with Ulcerative Colitis (UC). British Society of Gastroenterology guidelines recommend MTX in patients who are unresponsive or intolerant of thiopurines. Biologics are not available for UC because of local funding restrictions. Our practise is to only consider MTX in UC patients who have failed thiopurine therapy despite dose optimisation, including allopurinol co-therapy, to achieve therapeutic 6-thioguanine (TGN) and normal methylmercaptopurine nucleotide (MMPN) levels. To the best of our knowledge this is the first study to assess the clinical outcome of UC patients treated with MTX following thiopurine dose optimisation.

Methods Patients with UC treated with MTX were identified from a prospective IBD database. Outcomes following treatment with thiopurines were identified. All patients received MTX with folic acid supplementation. Patients were loaded with intramuscular 25mg MTX for 16 weeks followed by weekly maintenance MTX of $15 \mathrm{mg}$. Clinical response at 16 weeks and 12 months was used to assess the efficacy of MTX.

Results 9 patients (male $=8$ ) with UC treated with MTX were identified. Median age was 47.3 years (range; 24.8-77.8). Median time since diagnosis was 4.5 years (range; $1-25.5)$. Disease extent was extensive $(n=5)$, left sided $(n=1)$ and rectosigmoiditis $(n=3)$. Thiopurines had previously been discontinued in 9 patients because they were intolerant $(n=5)$ or unresponsive $(n=4)$ despite therapeutic TGN and normal MMPN levels.

2 patients (22\%) entered a steroid-free clinical remission with MTX at 16 weeks, which was sustained at 12 months. Both were intolerant of thiopurines because of severe nausea and dyspepsia. 7 patients (78\%) discontinued MTX at 16 weeks because of a lack of response $(n=6)$ or side effects ( $n=1$, pneumonitis). Clinical outcomes at 12 months for patients who failed MXT were colectomy $(\mathrm{n}=4)$, arsenic suppositories $(\mathrm{n}=2)$, and diagnostic reclassification $(\mathrm{n}=1)$ to Crohn's disease with CMV colitis treated with ganciclovir and infliximab.

Conclusion The efficacy of MTX in UC patients in this study was poor with a low clinical response rate $(22 \%)$ and high colectomy rate $(44 \%)$. Previous retrospective studies have reported MTX to be efficacious in patients failing thiopurines without reporting on the use of thiopurine optimisation. This study suggests that MTX has limited use in UC patients who are unresponsive to thiopurines despite dose optimisation.

Disclosure of Interest R. Basuroy: None Declared, H. Johnson Conflict with: Sponsorship from Falk, Abbott \& Warner Chilcott to attend meetings, T. Hollingworth: None Declared, S. Weaver Consultant for: MSD advisory board, Conflict with: Sponsorship from
Falk, Abbott, MSD \& Ferring to attend meetings, S. McLaughlin Conflict with: Sponsorship from Falk to attend meetings

\section{PTH-094 FAECAL CALPROTECTIN TESTING IN PRIMARY AND SECONDARY CARE - ARE THE CURRENT MANUFACTURER'S CUT-OFF LEVELS CLINICALLY USEFUL?}

doi:10.1136/gutjnl-2013-304907.581

1. ${ }^{*} \mathrm{~S} H$ Lee, ${ }^{1} \mathrm{H}$ Mainman, ${ }^{2} \mathrm{H}$ Borthwick, ${ }^{1,3} \mathrm{~A}$ Dhar. ${ }^{1}$ Gastroenterology; ${ }^{2}$ Clinical Biochemistry, County Durham \& Darlington NHS Foundation Trust, Bishop Auckland; '3School of Medicine and Health, Durham University, Stockton-on-Tees, UK

Introduction Faecal Calprotectin (FC) is a protein complex found in high concentrations in neutrophils and is released within the bowel when inflammation occurs. It can be measured quantitatively using ELISA and is very sensitive and specific in discriminating inflammatory from non-inflammatory pathologies causing diarrhoea. The manufacturer recommends a positive stool FC test $(>60 \mu \mathrm{g} / \mathrm{g}$ ) to be indicative of inflammation and further investigations (e.g. endoscopy, histology and imaging) and referral to a Gastroenterology clinic is advised.

Methods Aims: To determine whether the manufacturer's cut-off levels for referral $(>60 \mu \mathrm{g} / \mathrm{g})$ are clinically useful in making a positive diagnosis in patients presenting with chronic diarrhoea.Methods We analysed the outcome of $122 \mathrm{FC}$ test results done in primary and secondary care during a 3 month period from October to December 2011 performed on patients who presented with chronic diarrhoea without a pre-existing diagnosis of Inflammatory Bowel Disease (IBD). According to manufacturer's guidance, a FC result of $>60 \mu \mathrm{g} / \mathrm{g}$ was considered positive and $\leq 60 \mu \mathrm{g} / \mathrm{g}$ negative. Positive FC patients were seen in a Gastroenterology Clinic and investigated appropriately. The primary outcome of this study was to record the final diagnosis arising out of FC testing and investigations thereof. Secondary outcomes were to correlate FC levels to the final diagnosis.

Results Of $122 \mathrm{FC}$ tests, $41 \%(\mathrm{n}=51)$ were read as positive vs. $58 \%$ ( $n=71)$ negative. 19/51 (37\%) FC positive patients had a positive organic diagnosis $(\mathrm{IBD}=9$, Diverticulosis $=5$, Colonic Polyps $=3$, Infective colitis $=1$ and Chronic Pancreatitis =1) while the remaining 32 pts (63\%) were given a diagnosis of functional bowel disorder after investigations. Of 71 patients testing negative on $\mathrm{FC}, 94 \%$ $(n=67)$ had functional bowel disorder; only $6 \%(n=4)$ were found to have an organic condition, none of them IBD. This correlates with a positive predictive value of $37 \%$ and a negative predictive value of $94 \%$ for organic disease. The FC levels of those tested positive with a diagnosis of functional bowel disorder ranged from 61 $547 \mu \mathrm{g} / \mathrm{g}$ (mean $153 \mu \mathrm{g} / \mathrm{g}$ ) whereas FC values of those with organic conditions ranged from $63-1573 \mu \mathrm{g} / \mathrm{g}$ (mean $746 \mu \mathrm{g} / \mathrm{g}$ ).

Conclusion The current manufacturer's cut-off at $>60 \mu \mathrm{g} / \mathrm{g}$ is not clinically useful to diagnose an organic bowel pathology and further studies are needed to determine the true cut off value for a higher yield of a positive diagnosis. Cost effectiveness studies are also needed to determine referral cut off values.

Disclosure of Interest S. Lee: None Declared, H. Mainman: None Declared, H. Borthwick: None Declared, A. Dhar Grant/Research Support from: CLRN, NIHR, Speaker bureau with: Warner Chilcott UK, Shire Pharmaceuticals

\section{PTH-095 ARE IBD PATIENTS EITHER CRP PRODUCERS OR NON- PRODUCERS? A LONGITUDINAL STUDY}

doi:10.1136/gutjnl-2013-304907.582

1.*S T R Powles, 'A Varey, 'T Orchard, '1 Tyrrell-Price. 'Gastroenterology, Imperial College Healthcare NHS Trust, London, UK

Introduction C-reactive protein (CRP) is an established marker of disease activity in inflammatory bowel disease (IBD). However not all flares in IBD are associated with an elevated CRP. This raises the 
possibility that some patients are not CRP producers ${ }^{1,2,3}$. In this retrospective observational study, we have assessed if patients consistently produce a low or high CRP level in response to a flare in IBD. Methods 31 patients were identified, over a 5 year period across 3 centres, with endoscopic mucosal assessments of two consecutive exacerbations of IBD which were at least 3 months apart. Patients were included if they had a CRP measurement within 7 days of each endoscopic examination (colonoscopy or flexible-sigmoidoscopy), and if they had active inflammatory colitis confirmed on mucosal biopsy. A CRP non-producer was defined as a CRP level of less than $10 \mathrm{mg} / \mathrm{L}$ as according to the laboratory reference range used in the centres.

Results In the cohort of 31 patients, 19 had biopsy proven UC, 3 had Crohn's disease, 6 were unclassified, and 3 had differing classifications of Crohn's disease and UC on successive endoscopies. There was an overall mean period of 11.3 months between successive endoscopies. 17 patients were CRP non-producers and 9 were CRP producers during successive IBD flares. The table below shows the disease classification in different CRP response groups.

\section{Abstract PTH-095 Table 1}

\begin{tabular}{lccccc}
\hline & $\begin{array}{c}\text { Total number } \\
\text { of patients }\end{array}$ & UC & $\begin{array}{c}\text { Crohn's } \\
\text { disease }\end{array}$ & $\begin{array}{c}\text { Differing histological } \\
\text { classification }\end{array}$ \\
\hline CRP non-producer & 17 & 8 & 3 & 4 & 3 \\
CRP producer & 9 & 6 & 0 & 3 & 0 \\
Variable CRP response & 5 & 5 & 0 & 0 & 0 \\
\hline
\end{tabular}

Disease extent was defined in both flares in $97 \%$ of patients. In the CRP non-producer group, 10 out of 17 (59\%) patients had left sided disease or proctitis compared to 4 out of 8 (50\%) in the CRP producer group. This difference in proportion did not reach statistical significance as assessed by Fisher's exact test. Three of the five patients with a variable CRP response had more extensive disease at the time of the higher CRP level.

\section{Conclusion}

Most patients (84\%) in this study had a consistent CRP response; but this was not universal. Disease extent appears to contribute to CRP, but patient specific factors also appear to play a role.

Disclosure of Interest None Declared.

\section{REFERENCES}

1. Gut. 2008 Nov; 57(11):1518-23. Epub 2008 Jun 19. C-reactive protein: a predictive factor and marker of inflammation in inflammatory bowel disease. Results from a prospective population-based study. Henriksen M, et al. IBSEN Study Group

2. Inflamm Bowel Dis. 2005 Aug; 11(8):707-12..Correlation of C-reactive protein with clinical, endoscopic, histologic, and radiographic activity in inflammatory bowel disease. Solem CA, et al.

3. Inflamm Bowel Dis. 2004 Sep; 10(5):661-5.C-reactive protein as a marker for inflammatory bowel disease. Vermeire $\mathrm{S}$, et al.

\section{PTH-096 ORAL CICLOPSORIN IN STEROID REFRACTORY, ACUTE, SEVERE ULCERATIVE COLITIS}

doi:10.1136/gutjnl-2013-304907.583

1."S Gaggar, 'N Thompson. 'Gastroenterology, Freeman Hospital, Newcastle upon Tyne, Newcastle upon Tyne, UK

Introduction Ciclosporin (CsA) has been shown to be effective in treatment of steroid refractory severe ulcerative colitis and reducing the need for colectomy ${ }^{1}$. Most published evidence concerns intravenous infusions; however oral microemulsion ciclosporin (Neoral $\AA$ ) is well absorbed, more convenient and potentially less toxic. We report our experience with oral ciclosporin for treatment of steroid refractory severe ulcerative colitis.
Methods 30 consecutive patients receiving oral Ciclosporin for steroid refractory acute severe ulcerative colitis from October 2001 till July 2012 were retrieved from our clinical and pathology database. One patient received therapy twice. Hence, 31 episodes were analysed for this study.

Results $19 / 31$ patients were males. The median age at diagnosis and at the time of starting CsA was 39 years and 42 years respectively. 19 patients had pancolitis and 11 patients had left sided colitis. 4 patients were not on any treatment at the time of acute flare up. 28 patients were treated as inpatients. CsA was started after a median of 5 days after treatment with intravenous hydrocortisone in admitted patients (range 2-13 days). The mean CsA dose was $7.31 \mathrm{mg} / \mathrm{kg}$ on admission (range 5 to 8). The mean ciclosporin trough levels at 48-72hours and days $5-7$ were $167 \mathrm{ng} / \mathrm{ml}$ and $254 \mathrm{ng} / \mathrm{ml}$ respectively. The mean length of treatment was 23.6 weeks (range $1-123$ ). $50 \%$ had no side effects. One patient developed pyrexia of unknown origin necessitating stoppage of CsA. 26/31 (84\%) had initial clinical response. 5/31 had colectomies during the same admission while 15/31 (48\%) had colectomies within one year of starting ciclosporin treatment. 14/31 (46\%) have had no surgery till date after a mean follow up of 46 months (range 2-131). 14/26 who had initial response to CsA were started on azathioprine. Eleven were thiopurine naïve and out of these, $8 / 11(72 \%)$ are colectomy free till date. 12/26 were on thiopurines in the past. Only 4 of these 12 patients (33\%) are colectomy free till date.

Conclusion $84 \%$ of the cohort of patients having steroid refractory severe ulcerative colitis responded to CsA and 52\% retained their colon after 1 year. Our experience confirms CsA to be a safe drug with few side effects and should be used as a bridging therapy to azathioprine. Patients who are azathioprine naïve prior to CsA appear to have lower colectomy rates.

\section{Disclosure of Interest None Declared.}

\section{REFERENCE}

1. Campbell $S$, Travis $S$, Jewell D. Ciclosporin use in acute ulcerative colitis: a long term experience. Eur J Gastroenterol Hepatol 2005 Jan; 17(1):79-84

\section{PTH-097 DEFINING PATIENT-CENTRED PROFESSIONALISM IN GASTROENTEROLOGY}

doi:10.1136/gutjnl-2013-304907.584

1.*S Wright, ' J G Williams, 'H A Hutchings, 'F Rapport. 'College of Medicine, Swansea University, Swansea, UK

Introduction The UK IBD audit $3^{\text {rd }}$ round (RCP,2011)shows that there has been sustained improvement in patient-centred care in terms of clinician commitment to improved quality of care and the provision of specialist IBD nursing and patient education for patients with IBD. This study explores the meaning of the concept 'patient-centred professionalism' in gastroenterology. The objectives of this study are:

1. To clarify the meaning of patient-centred professionalism in terms of how it relates to professional practise and patientprofessional interaction in gastroenterological outpatient clinic consultations

2. To define the concept according to the views and experiences of healthcare professionals, stakeholders and IBD patients.

3. To create materials which may support and enhance optimal professional practise in outpatient clinics for patients with IBD

Methods A qualitative study using observation and semi-structured interviews. Ethnographic observation was conducted in 8 outpatient clinics from within one local health board (Abertawe Bro Morgannwg University Health Board). Clinics were led by consultant physician gastroenterologists; surgeons, specialist nurses and 